\title{
Arborescences
}

Revue d'études françaises

\section{Polyphonies : voix et valeurs du discours littéraire Introduction}

\section{Francis Langevin et Raphaël Baroni}

Numéro 6, septembre 2016

Polyphonies : voix et valeurs du discours littéraire

URI : https://id.erudit.org/iderudit/1037501ar

DOI : https://doi.org/10.7202/1037501ar

Aller au sommaire du numéro

Éditeur(s)

Département d'études françaises, Université de Toronto

ISSN

1925-5357 (numérique)

Découvrir la revue

Citer ce document

Langevin, F. \& Baroni, R. (2016). Polyphonies : voix et valeurs du discours

littéraire : introduction. Arborescences, (6), 1-12.

https://doi.org/10.7202/1037501ar

Tous droits réservés @ Département d'études françaises, Université de Toronto, Ce document est protégé par la loi sur le droit d’auteur. L’utilisation des 


\section{SOMMAIRE}

Francis Langevin et Raphaël Baroni.

01 University of British Columbia et Université de Lausanne Introduction

Alain Rabatel. Université de Lyon 1

13 L'énonciation problématisante : en dialogue avec

Le Royaume d'Emmanuel Carrère

Émilie Goin. Université du Luxembourg

39 Analyse d'un discours d'action collective mis en récit.

L'Anarchie dans Les Cloches de Bâle d'Aragon

David Bélanger. Université du Québec à Montréal

54 En contre-jour : la représentation évanescente de l'écrivain dans le roman québécois contemporain

Raphaël Baroni. Université de Lausanne

72 Comment débusquer la voix d'un auteur dans sa fiction?

Une étude de quelques provocations de Michel Houellebecq

Liesbeth Korthals Altes. Rijksuniversiteit Groningen

94 Actes de cadrage, narratologie et herméneutique - à propos de I'indétermination énonciative dans Sujet Angot de Christine Angot

121

Marion Sauvaire et Érick Falardeau. Université Laval

Susciter le moment critique. De l'investissement de ressources axiologiques

Frank Wagner. Université Rennes 2

148 Quand le narrateur boit(e)... (Réflexions sur le narrateur non fiable et/ou indigne de confiance) 


\title{
Polyphonies: voix et valeurs du discours littéraire
}

\author{
Francis Langevin. University of British Columbia \\ Raphaël Baroni. Université de Lausanne
}

50. Les voix des acteurs d'un vieux film muet sont doublées d'après les dialogues qu'ils ont réellement prononcés, que des sourds décryptent sur leurs lèvres. Le doublage n'a de rapport ni avec les images ni avec les intertitres.

—Édouard Levé (CEuvres. Paris : P.O.L. 2015 [2002] : 26)

C'est là que ça devient fou. Oui, c'est une des choses impossibles à démêler, comment un homme peut se laisser embarquer comme ça, sans presque résister à l'absurde, et ce chemin tracé à l'avance, humilié de plaisir. Ça fait partie, c'est vrai, des choses que je ne comprends pas, c'est comme des rails impossibles à quitter. Mais est-ce que ça me ferait dire un instant que c'en est un moins bon film, parce que quelque chose résiste à la compréhension, est-ce que ça me ferait dire que je n'ai pas trouvé ça formidable? Au contraire, d'autant plus formidable qu'il se permet d'incroyables omissions, et que, franchement, réussir à tenir un spectateur avec une telle absurdité, voilà un tour de force.

— Tanguy Viel (Cinéma. Paris : Minuit. 1999 : 38-39)

Le roman Cinéma de Tanguy Viel présente le monologue quasi inépuisable d'un homme qui a visionné compulsivement le même film, Sleuth (Mankiewicz 1972), et qui cherche à nous convaincre, comme tous ses amis avant nous, qu'il s'agit là d'un film formidable. On s'en doute, l'objet du roman, ce n'est plus tellement ce film, mais son spectateur et les interprétations qu'il produit. Euvres, d'Édouard Levé, décrit 533 œuvres potentielles, dont cette cinquantième, qui met l'accent sur une interprétation originale qui cherche à écouter une voix dans la fiction à laquelle personne n'avait encore prêté attention, mais qui est cependant lisible (du moins pour ceux qui savent lire sur les lèvres des acteurs). Cacophonies orchestrées par des lecteurs aux aptitudes discutables à travers des cadres incompatibles, ces fictions mettent en évidence des scripts énonciatifs plus complexes qu'ils n’y paraissent lorsque tout se passe sans incongruité. En effet, même les lectures les plus conventionnelles peuvent se caractériser par leur hétérogénéité lorsqu'on les compare avec celles produites par des "communautés 
interprétatives » différentes (pour reprendre un concept élaboré par Stanley Fish). Par exemple Céline mérite-t-il d'être lu ? Le Voyage au bout de la nuit est-il une œuvre antisémite ? Houellebecq a-t-il du style ? Soumission est-il un roman islamophobe? De nombreuses réponses contradictoires peuvent être apportées à ces questions essentielles sans que chacune des interprétations, prise isolément, ne paraisse dériver vers l'incohérence, l'excès ou la folie. L'apparent dysfonctionnement des interprétations les plus surprenantes ne fait donc que pointer la médiateté du récit et les rouages de son interprétation.

$\mathrm{Si}$ les récits de fiction peuvent potentiellement produire des interprétations aussi divergentes, et s'ils semblent avoir été programmés en partie pour cela, c'est entre autres en raison de leur indétermination énonciative et de la multiplicité des significations et des jugements de valeur qui en découlent. En effet, ainsi que le souligne Stanley Fish, n'importe quel énoncé, même l'assertion la plus banale, peut déboucher sur une pluralité interprétative que l'on peut rapprocher du concept de dialogisme chez Bakhtine.

Quand un homme dit à sa femme : "ce n'est pas toi, c'est ta mère qui
parle», il témoigne, premièrement, du fait que les significations que
l'on attribue aux mots varient suivant le locuteur auquel ils sont
assignés et, deuxièmement, du fait que les mots ne vous disent pas qui
est ce locuteur. (Fish $2007: 135$ )

On notera que dans le cas mentionné par Fish, le problème n'est pas limité au marquage linguistique de la polyphonie - ce que Jacqueline Authier-Revuz (1982) appellerait l'hétérogénéité montrée du discours - mais inclut également une hétérogénéité constitutive, propre à la nature même du langage humain. En effet, tout énoncé peut être rattaché à une instance énonciative différente de la personne qui l'articule sans que le texte et le contexte - qui certes orientent l'interprétation - suffisent à confirmer de manière absolue cette attribution : la femme pense probablement s'exprimer en son nom propre, mais son mari n'est pas de la même opinion, car il prétend avoir déjà entendu ces paroles ailleurs. Ainsi, l'hétérogénéité du discours dépend autant, en amont, de l'origine dialogique de la parole, qu'en aval, de la manière plus ou moins imprévisible dont elle sera interprétée dans un contexte singulier. Ce qui, dans la conversation et les communications quotidiennes, débouche souvent sur des malentendus plus ou moins malheureux, produit au contraire, en littérature, de riches problématisations. Car il faut rappeler que le récit fictionnel est d'emblée placé sous le signe de la complexité et de l'équivoque, ce qui a amené Umberto Eco à décrire le roman comme une "machine paresseuse" (1985: 29) qui requiert, pour signifier quelque chose, la coopération d'un lecteur plus ou moins modèle, ou au contraire rebelle. Et l'hétérogénéité des interprétations se vérifie non seulement dans la manière de 
se représenter les mondes possibles de la fiction, mais surtout dans la manière de percevoir le dispositif énonciatif du récit. En effet, ainsi que le relève Alain Rabatel :

Le sujet racontant, par cela même qu'il raconte, et surtout par le fait même de raconter, en mettant en scène des centres de perspective différents, ouvre potentiellement une boîte de Pandore d'où sortent des voix autorisées et d'autres qui le sont moins, mais qui néanmoins sapent l'autorité des premières, en sorte que le récit, loin d'être l'illustration d'une vérité prétablie, ouvre sur les possibles infinis de l'interprétation. (Rabatel $2009: 17$ )

Il est certainement impossible de déterminer, de manière objective et définitive, la voix authentique qui se dissimule derrière tel ou tel énoncé fictionnel, mais il ne s'agit pas non plus d'affirmer, à l'instar de Roland Barthes (1986 : 63), que l'écriture serait «destruction de toute voix » et « de toute origine ". En effet, pour donner du sens au discours, l'interprète relie spontanément les énoncés fictionnels à des instances qui pourraient en être tenues responsables (auteur, narrateur, personnage, doxa, vérité, etc.) et c'est la valeur du texte qui se joue au moment de cette attribution déterminante. Dans le cas de la fiction, ainsi que l'a montré Wayne C. Booth (1977 : 9293), on peut toujours soupçonner que l'auteur, en tant que producteur implicite de l'énoncé, se cache derrière l'instance fictionnelle qui est censée assumer le discours : "Même un roman dans lequel aucun narrateur n'est représenté suggère l'image implicite d'un auteur caché dans les coulisses, en qualité de metteur en scène, de montreur de marionnettes, ou comme dit Joyce, de dieu indifférent curant silencieusement ses ongles ». C'est ce qui faisait dire à Platon que le poète se camoufle "lorsqu'il rapporte un discours particulier comme s'il était quelqu'un d'autre " (République, livre III, 393b-c) et l'on se souvient de la conséquence dramatique, sur le plan politique, que le philosophe tirait de cette manière indirecte de faire usage du langage. Ainsi, entendre l'ironie d'un auteur implicite derrière la description apparemment objective du flux de conscience d'un personnage immoral ou au contraire voir dans ce personnage un porte-parole de l'écrivain, cela peut avoir des conséquences non seulement esthétiques, mais également éthiques, voire juridiques; ce n'est pas Flaubert qui dira le contraire, ni Houellebecq, d'ailleurs. Dominique Maingueneau (2004) rappelle quant à lui que la "scénographie " romanesque est toujours une "mise en scène ", construite par l'auteur en vue de légitimer son discours. Dans ce concert polyphonique des voix romanesques, il s'agit donc de s'interroger sur les stratégies discursives (scénographie, éthos) et contextuelles (posture publique, paratextualité, etc.) mobilisées par l'auteur dans l'espoir de canaliser le sens de l'œuvre, stratégie qu'il faut pouvoir également contraster avec les 
interprétations des lecteurs empiriques ou des communautés de lecteurs qui se dessinent à travers l'histoire de la réception.

Dans ce numéro nous avons donc souhaité nous intéresser à ce lieu de rencontre entre les questions relatives à la polyphonie énonciative et la négociation des valeurs qui en découle dans le récit de fiction. Si la polyphonie peut être envisagée - en raison de la tradition de lecture engendrée à partir de la notion bakhtinienne du dialogisme - comme la confrontation d'idéologies et de voix concurrentes, elle est aussi l'occasion, ainsi que le suggère Alain Rabatel (ce numéro), de "mettre l'accent sur la multiplication des points de vue et la complémentarité des perspectives pour penser le complexe". Pour le linguiste, qui s'intéresse depuis longtemps à la question du point de vue dans l'énonciation, il s'agit ici moins de s'adonner à un inventaire des voix que d'aborder le rapport qu'entretiennent points de vue, vérité et valeurs, "sans céder au relativisme ni au sectarisme ». Face à une énonciation problématisante, c'est-à-dire lorsque le locuteur (source de la voix) et l'énonciateur (source du point de vue) ne forment pas une mais plusieurs combinaisons qui sont l'objet d'autant d'hypothèses interprétatives, Rabatel place l'empathie au cœur de sa lecture. Le Royaume, roman d'Emmanuel Carrère (2014), est entièrement consacré à l'interprétation. Le Carrère non croyant actuel relit le Carrère croyant du passé au moyen d'un récit qui raconte, à partir d'une copieuse recherche, les premiers temps de la chrétienté. Carrère suit l'apôtre Paul et l'évangéliste Luc - et c'est cette démarche qui alimente la quête interprétative. La lecture d'Alain Rabatel aborde ce roman en suivant les actes d'interprétation empathiques représentés, mais aussi ceux qui sont utiles à l'interprétation du roman lorsque l'énonciation se fait problématisante; il fait valoir la pertinence de la question des points de vue, qui permet au lecteur d'articuler voix et valeurs en "reconstructions hypothétiques" qui s'enrichissent de la polyphonie. La construction délibérément subjective de l'objet du roman est une façon pour Carrère de "penser son rapport complexe à l'altérité ", affirme Alain Rabatel ; cela est également visible quand le romancier "déplace" ou décentre son regard sur l'objet et modifie les modalités de son empathie. Cette "grande diversité des mouvements empathiques " est à la fois construite et montrée : elle est montrée par le récit qu'élabore Carrère des diverses reconstructions imaginaires, mais plausibles, des motivations de Luc, par exemple, mais elle est aussi construite par la variabilité du point de vue dans le récit, qui assemble, commente et croise les témoignages. Se pose essentiellement la question de la vérité de la parole de Jésus, rapportée par de multiples intermédiaires. L'enjeu, après tout, est celui de la croyance, et c'est la croyance qui fait exister son objet et les conditions mêmes de la croyance. Il est là, le Royaume. Le roman, lui, pose essentiellement cette question à l'ensemble des "scènes énonciatives" que le narrateur Carrère assemble en les observant selon des perspectives diverses, en développant pour elles des points de vue (voix + point de vue) qui nourrissent une réflexion polyphonique prenant 
appui sur des énonciations problématisantes. Cette réflexion dialogique sur les valeurs, la vérité et la relativité permet en définitive de "mieux entrer dans la complexité des choses ", écrit Alain Rabatel, dont la posture interprétative, comme celle de Carrère dans Le Royaume, s'apparente à l'éthos de Luc, puis à celui de Jésus que dessine petit à petit le romancier : "figure d'un honnête homme toujours en recherche, qui prend en compte la relativité des choses, des vérités, et s'oblige à d'incessantes, inconfortables synthèses ". C'est aussi la conciliation des contraires, une ouverture aux "plusieurs manières d'accéder à Dieu, au salut, à la vérité, à la sagesse ".

Si Le Royaume se présente d'emblée comme un foisonnement heureux de pistes interprétatives au sein d'un cadre qui en relance les énergies divergentes, le roman Les Cloches de Bâle (1934) de Louis Aragon, abordé dans une perspective énonciative par Émilie Goin, ne jouit pas de la même réputation. L'étiquette de "roman à thèse " pèse sur l'interprétation de ce roman jugé trop esthétique par le Parti communiste et trop politique par la critique littéraire... Goin prend le parti d'explorer le dispositif du collage qui, dans le roman d'Aragon, donne lieu à un déplacement axiologique du cadre interprétatif initial. La transposition étudiée est la citation (sans trace), dans le discours d'un personnage, du texte de l'annonce d'un meeting, parue en 1906 dans le journal L'Anarchie. L'article d'Émilie Goin montre que la radicalité du positionnement politique du journal (anarchiste et antisyndicaliste, négation de la lutte des classes) d'où est tirée cette annonce, est en contradiction avec ce qu'elle annonce: une mobilisation. Le personnage de Libertad, dans Les Cloches de Bâle, devient le locuteur individuel d'un discours collectif qui s'exprime à travers lui. L'analyse fine des points de vue de Libertad se déroule au croisement de contradictions de voix et de valeurs qui sont ici mises en relation avec l'image de l'écrivain Aragon qui, au début des années 1930, " cherche à rompre avec son passé surréaliste et anarchiste pour s'engager dans le communisme». Si la mise en récit des points de vue de Libertad illustre une critique du discours anarchiste, elle présente aussi un éthos qui n'est pas sans rappeler la posture d'Aragon lui-même, parfois ambivalente, et du programme politique et esthétique du réalisme socialiste qu'il théorise et souhaite mettre en pratique.

Jérôme Meizoz a bien décrit ces postures qui sont aptes à modifier l'interprétation des textes: externes à l'œuvre, elles résultent d'une "présentation de soi dans des contextes où la personne incarne la fonctionauteur"; internes à l'œuvre, elles sont le résultat d'une "construction de l'image de l'énonciateur dans et par le texte" (Meizoz 2007: 23). Tant Emmanuel Carrère que Louis Aragon jouissent d'un éthos d'écrivain qui les précède et que leur œuvre nourrit, ces rôles préconstruits et toujours en construction. C'est parce qu'elles peuvent être plurivoques que ces postures retiennent l'attention, qu'elles peuvent générer une adhésion et qu'elles peuvent aussi pointer en direction des valeurs. Qu'il s'agisse de fictions ou des formes variées de l'autobiographie ou de l'essai personnel, les stratégies 
rhétoriques de présentation de soi portent avec elles les effets qu'elles souhaitent engendrer. Ces régimes d'auctorialité sont sans cesse en jeu dans la narration, comme l'écrit Ruth Amossy dans sa définition de l'éthos : «la posture qu'adopte le narrateur à travers les modalités de sa parole écrite module sa relation avec le lecteur et sa capacité à l'influencer et l'émouvoir. " (Amossy 2002 : 201)

David Bélanger s'intéresse quant à lui à des figures délibérément fictives de l'écrivain : l'écrivain négatif (qui n'écrit pas) et l'écrivain frauduleux (qui n'a pas écrit), parties d'une posture - ou d'une paratopie (Maingueneau 2004) - plus vaste : celle de l'anti-écrivain dont l'auctorialité, au surplus, est mise en doute. L'examen de ces trois types de posture permet à Bélanger de présenter à nouveaux frais un panorama critique des représentations du romancier dans le roman québécois contemporain. Dans Une estafette chez Artaud de Nicolas Tremblay (2011), la multiplication des récits impersonnels et paratextuels semble viser l'effacement de l'écriture du personnage « Nicolas Tremblay ", et constituerait une "autogénèse littéraire négative " (Bélanger, ce numéro). Pleurer comme dans les films de Guillaume Corbeil (2009), deuxième roman abordé par Bélanger, pourrait être rapproché du Quichotte de Pierre Ménard (1939) et de Pseudo d'Émile Ajar (1976) : le narrateur personnage se projette dans une écriture qui n'est jamais la sienne, et qui est chaque fois conditionnée par un désir de "faire écrivain ". Ces jeux sur la figure $\mathrm{du}$ romancier, sur les écrivains imaginaires (effacement, surdétermination, renversement) remettent en question le statut de l'auteur et de la littérature - parmi les discours. Pour ces écrivains contemporains, désigner les zones frontalières de ce discours (le roman québécois) en montrant le procès d'attribution des voix et des valeurs du romancier, produit paradoxalement une contribution propre à nourrir ce que l'on tente d'invalider en s'y inscrivant, en s'y positionnant, en proposant des scénographies limites, etc. Le moyen d'y échapper? Ce non-discours sur l'auctorialité qui produit une méta-auctorialité nous invite à revenir à la notion de discours constituant présentée par Dominique Maingueneau et Frédéric Cossutta, qui écrivaient à ce sujet : «l'énonciation se déploie comme dispositif de légitimation de l'espace de sa propre énonciation, à l'articulation d'un texte et d'une manière de s'inscrire dans un univers social " (Maingueneau et Cossutta 1995: 115). Ainsi, la littérature, le discours littéraire et le roman en particulier ne sauraient, semble-t-il, échapper à cette circularité de l'institution. L'autorité discursive du discours littéraire, selon Dominique Maingueneau, dépend de ce qu'il appelle une " paratopie », c'està-dire une "appartenance paradoxale, qui rend possibles des énonciations 
prétendant excéder l'espace qu'elles ont pour fonction de fonder $»{ }^{1}$ Dans Le discours littéraire, il insiste sur la productivité du paradoxe de l'auctorialité :

Condition de l'énonciation, la paratopie de l'écrivain en est aussi le produit ; c'est à travers elle que l'œuvre peut advenir, mais c'est aussi elle que cette œuvre doit construire dans son développement même. Énonciation foncièrement menacée, la littérature ne peut dissocier ses contenus de la légitimation du geste qui les pose, l'œuvre ne peut configurer un monde que si ce dernier est déchiré par le renvoi à l'espace qui rend possible sa propre énonciation. (Maingueneau 2004 : 94)

Dans leurs contributions respectives, Raphaël Baroni et Liesbeth Korthals Altes s'interrogent quant à eux sur la place qu'occupe la figure de l'auteur dans le texte et hors du texte - dans l'interprétation d'œuvres dont la réception fait polémique. Baroni montre que le lecteur des romans de Michel Houellebecq - qui a fait de la provocation, ou du moins du "politiquement incorrect ", sa marque de fabrique - peut toujours soupçonner que derrière les propos (plus ou moins scandaleux) tenus par les personnages romanesques se dissimule une origine auctoriale n'échappant pas, elle, à une évaluation éthique. Cela est d'autant plus important que la valeur esthétique de l'œuvre est directement corrélée à la manière dont elle s'inscrit dans les valeurs de son époque. Chez Houellebecq, en raison de la confusion opérée entre les voix des personnages, des narrateurs et de l'écrivain, la frontière est poreuse qui sépare "les opinions des personnages de celles de l'auteur, au risque de renforcer l'écrasement de la polyphonie", écrit Baroni. En examinant différentes " provocations » que l'on rencontre dans Les Particules élémentaires (1998), il suggère cependant qu'en dépit de l'indétermination énonciative qui caractérise les énoncés fictionnels, l'auteur peut tenter de canaliser l'interprétation en recourant à différentes stratégies textuelles et contextuelles. En d'autres termes, en jouant sur des scénographies différenciées, certaines provocations fictionnelles apparaîtraient plus ou moins assumées par un auteur pas aussi implicite qu'on ne le croirait d'emblée, puisqu'il peut également commenter publiquement ses romans, voire reprendre à son compte tel ou tel discours fictif, ou au contraire s'en distancer. Néanmoins, ce dispositif ne permet pas de canaliser totalement la lecture, et d'autres «cadres interprétatifs ", pour utiliser une notion issue des sciences sociales, demeurent possibles.

C'est justement sur l'opérativité de cette notion de "cadrage " pour une réflexion métaherméneutique (c'est-à-dire pour l'étude de la manière

${ }^{1}$ Définition tirée du glossaire que l'on trouve sur le site de Dominique Maingueneau. URL : http://dominique.maingueneau.pagesperso-orange.fr/glossaire.html\#Para. Page consultée le 16 août 2016. 
dont nous interprétons les textes) que se concentre l'article de Liesbeth Korthals Altes. Comme dans les analyses que propose Rabatel, Korthals Altes éclaire la multiplication des instances d'énonciation - de leurs voix et de leurs choix, si l'on veut - qui problématisent l'interprétation d'un roman : Sujet Angot de Christine Angot (1998). L'œuvre, polémique elle aussi, sert de terrain de discussion pour présenter les thèses défendues dans Ethos and Narrative Interpretation (2014), où Korthals Altes explore les linéaments de ce " don particulier d'entendre et de comprendre toutes les voix ensemble " que reconnaît Bakhtine à Dostoievski (Bakhtine 1970 : 64). Elle se penche ici sur le rôle joué par l'éthos (de l'écrivain, du narrateur) dans la négociation des valeurs au sein du discours de fiction, en proposant l'hypothèse " qu'en cas de brouillage ou de dilemme de cadrage générique et énonciatif, le lecteur se réfère, entre autres, à l'éthos et à la posture qu'il pense pouvoir attribuer à l'auteur» (Korthals Altes, ce numéro). L'interprétation des textes où le statut des voix est problématique place le lecteur au cœur d'interactions entre des cadres interprétatifs qui dépassent la littérature. Cette herméneutique convoque d'autres cadres nécessitant de la lecture calculs, positionnements et "actes de cadrage» personnels et collectifs divers, informés par d'autres situations d'énonciation, en particulier en face de " points de vue idéologiques ou éthiques controversés dans l'opinion ». Voix narratives (leur imbrication, leur crédibilité), points de vue, genre (fiction ou référentialité ? autobiographie ou autofiction ?), posture d'auteur (ironie ou sincérité ?) : ces cadrages issus des théories littéraires sont mis en face des "régimes de valeurs qu'ils impliquent conventionnellement». Les fictions dont les voix et les valeurs font procès et relancent l'interprétation désignent, selon Korthals Altes, la direction que pourrait prendre les recherches narratologiques :

\footnotetext{
À la lumière de ce que l'on comprend des mécanismes de la production du sens et des valeurs grâce à la sociologie, à la sémiotique et aux sciences cognitives, il semble plus prometteur et judicieux de s'attacher à reconstituer les schémas, en grande partie conventionnels, selon lesquels des lecteurs font sens à partir des textes.
}

C'est sur ces "sujets lecteurs" que se penchent Marion Sauvaire et Érick Falardeau dans leur article, qui présente les résultats d'une étude empirique visant à observer quelles ressources axiologiques des élèves de lycée (France) et de cégep (Québec) mettent en œuvre pour interpréter la nouvelle fantastique "La plage des songes» de Stanley Péan (1998). Il s'agit plus précisément d'observer comment ces lecteurs en formation "prennent conscience réflexivement du caractère subjectif de leur lecture et en particulier des présupposés idéologiques et axiologiques qui influencent leurs interprétations ". Cette compétence "métaherméneutique ", pour reprendre le terme employé dans ce numéro par Korthals Altes, est analysée dans des textes réflexifs rédigés par les élèves. Les travaux antérieurs de Sauvaire (2013) 
avaient déjà montré l'importance de l'accès à des cadres interprétatifs variés dans l'interprétation des textes littéraires: " les élèves qui produisent plusieurs interprétations riches et complexes sont ceux qui mobilisent des ressources de différents types" (Sauvaire et Falardeau, ce numéro). La nouvelle de Péan s'inscrit au croisement des diverses poétiques de l'écriture caraïbe québécoise et de l'indécidabilité caractéristique du fantastique. Elle mobilise chez les lecteurs en formation des ressources axiologiques en raison de quelques défis interprétatifs liés aux valeurs motivant l'action du protagoniste (Christian) et à la crédibilité du point de vue de la narratrice (Évelyne). Or, de façon assez importante pour ce numéro, Sauvaire et Falardeau montrent que «[p]our construire du sens, les élèves relient assez spontanément des énoncés à des instances qui en seraient responsables, l'auteur au premier rang ». Outre cette interprétation fondée sur une recherche des intentions par un cadrage auctorial (biographie de l'écrivain) et une identification aux personnages, où la direction de l'œuvre et les motivations des personnages sont unies par les notions d'intentionnalité et d'unité, les lecteurs en formation recourent aussi à des ressources axiologiques personnelles (jugements, valeurs, comportements exemplaires) afin de donner sens aux actions des personnages. L'apprentissage de la lecture littéraire " offre une médiation privilégiée pour mettre à distance de manière critique les normes et les valeurs plus ou moins partagées par une communauté interprétative ". Autrement dit, l'activité réflexive propre à ce cadrage énonciatif - la lecture littéraire - suscite un "moment critique " de mise à distance du texte et une prise de conscience de l'hétérogénéité énonciative; la lecture de l'hétérogénéité énonciative permet également aux apprenants d'appréhender les valeurs en circulation dans l'univers social, audelà de leur propre investissement subjectif ou de l'intentionnalité pure.

On ne doit pas se surprendre que de jeunes lecteurs recourent à la figure de l'auteur et à leurs propres régimes de valeurs pour faire sens de textes dont les enjeux éthiques sont problématiques. La narratologie s'y égare ellemême lorsque vient le temps de décrire le récit en suivant un modèle communicationnel, en particulier en présence de narrateurs non fiables ou indignes de confiance. La réflexion que présente Frank Wagner, en clôture de ce numéro, propose un état des lieux des diverses approches de l'" unreliable narrator", relativement peu discutées dans la critique francophone. Wagner explore les dimensions sémiotique et pragmatique des critères de repérage, de "diagnostic » et d'interprétation de la non fiabilité narrative. Le narrateur non fiable ou indigne de confiance se trouve en effet à la croisée de la polyphonie (qui parle ?) et des valeurs (qui pense ?), notamment parce que c'est la valeur du point de vue exprimé qui fait procès. Cet état des lieux nous guide au cour du désaccord entre les tenants d'une narratologie immanente et textualiste, pour laquelle les cadres interprétatifs sont des représentations textuelles de la communication supposées déclencher des effets déterminés, et les approches dites cognitives, pour lesquelles les cadres interprétatifs englobent plus largement l'expérience de lecture elle-même. Les théories de 
l'effet et les théories de la réception correspondent à deux démarches distinctes, selon Wagner: repérer des indices textuels de non fiabilité et étudier la façon dont les lecteurs réagissent à ces "signaux " en fonction de leurs valeurs. Suivant cette approche cognitive, le narrateur non fiable ou indigne de confiance serait alors non pas un effet du texte (Jouve 2001), mais une stratégie interprétative disponible, une posture ou une instance mise en œuvre par la lecture, ce qui n'est pas sans rappeler le décodage de l'ironie (A. Nünning 1999). Qu'elle soit envisagée comme une mention ou comme un simulacre, c'est parce que la figure de l'ironie est disponible dans le contexte qui la voit naître qu'elle peut faire partie des hypothèses interprétatives. Après un examen de diverses typologies des figures de narrateur non fiable et/ou indigne de confiance (Mercier et Fortier 2011 ; Langevin 2011), Wagner suit la piste de l'ironisation - un surplomb des voix - qui peut survenir et être repérée plus aisément chez les narrateurs homodiégétiques, garants de leurs propres voix. Mais que faire des narrateurs hétérodiégétiques indignes de confiance, sinon recourir à la notion de l'auteur impliqué, ${ }^{2}$ cette image de l'auctorialité projetée sur une énonciation dite impersonnelle qui dépend de la bienveillance et de la perspicacité des lectrices et lecteurs? Séduire et éveiller le lecteur : il pourrait s'agir là, écrit Wagner, de la convergence des fonctions du narrateur non fiable dans la littérature contemporaine. Ces indéterminations sont responsables d'accrochages dans le discours sur les œuvres des écrivains, elles jouent de cette zone franche où se brouillent l'origine des voix et leur vectorialité. L'étude des divergences interprétatives diachroniques (V. Nünning 2004) et synchroniques (Bayard 1998) que présente Wagner pointe en direction du relativisme dont souhaite aussi se garder Alain Rabatel dans l'article qui inaugure ce numéro. Tant Rabatel, Baroni, Korthals Altes que Wagner mettent en ouvre le mode de lecture bakhtinien, qui consiste à maintenir active l'indétermination des intentions de l'œuvre, la possibilité qu'entre le projet, sa réalisation et ses interprétations, il puisse y avoir un espace où le lecteur doit faire des choix.

Les lieux d'indéterminations identifiés par les collaboratrices et les collaborateurs de ce numéro consacré à la polyphonie ont tous ceci de commun qu'ils conditionnent "un espace interprétatif en tension " (Baroni 2014 : \$17), un "espace d'échange" inclusif ou exclusif (Langevin 2009: $\$ 21)$ où l'expérience de lecture est loin d'être une activité passive. Le lecteur est plutôt invité à construire sa propre Komplementärgeschichte (Stanzel 2004 : 203) autour de l'attribution des voix, des points de vue et des valeurs. Pris dans les fils de l'intrigue, assujettis à des "cadres herméneutiques " (Maingueneau 2004: 56), nous engendrons une "fiction herméneutique "

\footnotetext{
${ }^{2}$ En français, l' «implied reader » de Booth est tantôt traduit par " auteur implicite » (ce qui semble être la traduction la plus littérale, et c'est d'ailleurs le terme que l'on trouve dans le seul article de Booth publié en version française dans la revue Poétique) tantôt par "auteur impliqué " (terme utilisé notamment par Genette dans Nouveau discours du récit).
} 
(Langevin 2015 : 51) où l'enjeu est un double positionnement : nous devons d'abord déterminer qui parle, et selon quels points de vue. L'acte interprétatif, dès lors, nous engage : nous devons nous révéler, nous devons prêter attention " aux présupposés qui structurent nos interprétations" (Wagner, ce numéro) lorsque nous choisissons d'entendre telle ou telle voix ou d'adopter tel ou tel point de vue.

\section{Références bibliographiques}

Amossi, R. 2002. "Ethos". Dans Dictionnaire du littéraire, sous la direction de P. Aron, A. Viala et D. Saint-Jacques. Paris : Presses Universitaires de France : 200-201.

Authier-Revuz, J. 1982. "Hétérogénéité montrée et hétérogénéité constitutive : éléments pour une approche de l'autre dans le discours ", DRLAV 26:91-151.

Bakhtine, M. 1970. La poétique de Dostoievski. Paris : Seuil.

Baroni, R. 2014. «La guerre des voix. Critique polyphonique et divergences interprétatives dans l'œuvre de Michel Houellebecq ". COnTEXTES 4. URL : http://contextes.revues.org/5979. Page consultée le 3 août 2016.

Bayard, P. 1998. Qui a tué Roger Ackroyd ? Paris : Minuit.

Booth, W. C. 1968. The Rhetoric of Fiction. Chicago : University of Chicago Press.

Booth, W. C. 1977. "Distance et point de vue. Essai de classification ». Dans Poétique du récit, sous la direction de G. Genette et T. Todorov. Paris : Seuil : 85-113 [1961].

Eco, U. 1985. Lector in Fabula. Paris : Grasset.

Fish, S. 2007. Quand lire c'est faire. Paris: Les prairies ordinaires.

Genette, G. 1983. Nouveau discours du récit. Paris : Seuil.

Hamon, P. 1996. L'ironie littéraire. Essai sur les formes de l'écriture oblique. Paris: Hachette.

Jouve, V. 2001. "Qui parle dans le récit?" Cahiers de narratologie 10 (2) : 75-90.

Korthals Altes, E. J. 2014. Ethos and Narrative Interpretation: The Negotiation of Meanings and Values in Fiction. Lincoln : University of Nebraska Press.

Langevin, F. 2009. "La connivence construite par le discours de l'évidence. Attitude du narrateur et vraisemblance chez Patrick Lapeyre et Jean Echenoz». Temps zéro 2. URL : http://tempszero.contemporain.info/document384. Page consultée le 3 août 2016.

Langevin, F. 2011. "La posture exotique du narrateur-personnage. Inconfort et non-fiabilité dans quelques romans contemporains". Dans La transmission narrative. Modalités du pacte romanesque contemporain, sous la direction de F. Fortier et A. Mercier. Québec: Nota Bene : 207-233.

Langevin, F. 2015. Lire le narrateur. Tensions de la narration hétérodiégétique dans le roman contemporain. Montréal : Nota Bene.

Maingueneau, D. 2004. Le discours littéraire. Paratopie et scène d'énonciation. Paris : Armand Colin.

Maingueneau, D. et F. Cossutta. 1995. "L'analyse des discours constituants ». Langages 29 (117) : 112-125.

Meizoz, J. 2007. Postures littéraires. Mises en scène modernes de l’auteur. Genève : Slatkine.

Mercier, A. et F. Fortier. 2011. "La narration impossible. Conventions réalistes, catégories narratologiques et enjeux esthétiques ". Dans La transmission narrative. Modalités du pacte romanesque contemporain, sous la direction de F. Fortier et A. Mercier. Québec: Nota Bene : 333-356.

\section{Arborescences}

Revue d'études françaises

ISSN: 1925-5357 
Nünning, A. F. 1999. "Unreliable, Compared to What? Towards a Cognitive Theory of Unreliable Narration: Prolegomena and Hypothesis." Dans Grenzüberschreitungen: Narratologie in Kontext / Transcending Boundaries: Narratology in Context, sous la direction de W. Grünzweig et A. Solbach. Tübingen : Gunter Narr Verlag : 53-73.

Nünning, V. 2004. "Unreliable Narration and the Historical Variability of Values and Norms: The Vicar of Wakefield as a Test-case of a Cultural-Historical Narratology." Style XXXVIII-2 : 236-252.

Rabatel, A. 2009. Homo Narrans. Pour une analyse énonciative et interactionnelle du récit. Tome 1 : Les points de vue et la logique de la narration. Limoges : Lambert-Lucas.

Sauvaire, M. 2013. Diversité des lectures littéraires. Comment former des sujets lecteurs divers? Thèse de doctorat. Québec et Toulouse: Université Laval et Université Toulouse-leMirail.

Stanzel, F. K. 2004. “The 'complementary story': outline of a reader-oriented theory of the novel." Style $38: 2: 203-220$. 